\title{
Minimally Invasive Fixation of Anterior Pelvic Ring Disruption Using INFIX Technique
}

ISSN: 2576-8875

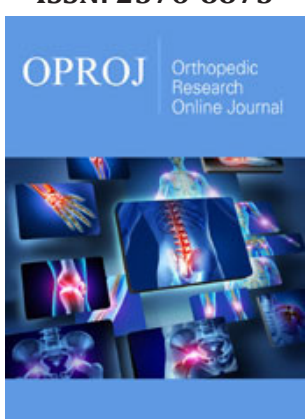

*Corresponding author: Ebeed Yasin, Aswan University, Egypt

Submission: 每 February 07, 2020

Published: 想 February 18, 2020

Volume 6 - Issue 5

How to cite this article: Ebeed yasin MD, Faisal Adam MD, Hesham Refae MD, Mahmoud Abdelgaliel MSc, Ahmed Abdellatif MD, Morsy Basiony MD, Mohamed Salman MD. Minimally Invasive Fixation of Anterior Pelvic Ring Disruption Using INFIX Technique. Ortho Res Online J. 6(5). OPROJ.000646.2020.

DOI: 10.31031/OPROJ.2020.06.000646

Copyright@: Ebeed Yasin, This article is distributed under the terms of the Creative Commons Attribution 4.0 International License, which permits unrestricted use and redistribution provided that the original author and source are credited.

\author{
Ebeed yasin MD ${ }^{1 *}$, Faisal Adam MD $^{2}$, Hesham Refae MD ${ }^{1}$, Mahmoud Abdelgaliel \\ MSc $^{1}$, Ahmed Abdellatif MD ${ }^{1}$, Morsy Basiony MD ${ }^{1}$, Mohamed Salman MD ${ }^{1}$ \\ ${ }^{1}$ Orthopaedic Department, Aswan University, Egypt \\ ${ }^{2}$ Orthopaedic Department, Assiut University Hospital, Egypt
}

\begin{abstract}
Background: The posterior pelvic ring provides the main stability $60 \%$, the anterior ring account for $40 \%$ of stability of the pelvic ring. Unstable pelvic ring injuries usually associated with high energy trauma and high mortality and morbidity. External fixation is commonly used as a definitive fixation for unstable anterior pelvic ring fractures, but it has many drawbacks. INFIX, recently developed as minimally invasive technique utilized by authors to achieve stable fixation and good patient satisfaction with minimal complication.
\end{abstract}

Purpose: The preliminary study aimed to evaluate the fracture reduction, stability, bone healing, intra and postoperative complications using the INFIX technique.

Methods: A prospective cohort study was carried out for patients with unstable anterior pelvic ring fractures in Aswan University Hospital and Assiut University Hospital utilizing spinal pedicle screws inserted into the supra-acetabular bone and connected by a subcutaneous rod (INFIX).

Material: Sixteen patients were carried out with one year follow up, (62.5\%) pelvis C and (37.5\%) pelvis B fracture. (94.75\%) males and (6.25\%) females their age ranges between (17-43 years), Mean (29.54 \pm 13.6$)$.

Results: According to radiological Matta criteria, (81.25\%) excellent reduction, (12.5\%) good reduction and (6.25\%) fair reduction. According to Majeed Score, $(93.75 \%)$ excellent and (6.25\%) good clinical outcome. Postoperative complications, 3 cases of lateral femoral cutaneous nerve (LFCN) irritation (18.75\%) and 2 cases of asymptomatic heterotopic ossification $(12.5 \%)$

Conclusions: INFIX is a reliable method for fixation of anterior pelvic ring with small incision, short time for application, excellent clinical and radiological outcome with fewer complications.

Keywords: Pelvis; Fractures; Anterior ring; INFIX

\section{Introduction}

Management of high energy pelvic ring fractures represents a challenging issue for orthopedic surgeons. Unstable high energy pelvic ring fractures usually associated with high morbidity and mortality [1,2]. Urgent intervention with simple stable fixation device is needed to achieve hemodynamic stabilization. External fixation is commonly used for fixation of unstable anterior pelvic ring fractures. Pin site infection, fixation loosening, patients unsatisfied, nerve injury, delayed rehabilitation and difficult nursing are common complications with external fixator [3]. Open reduction and internal fixation using plate need more dissection in dangerous area and associated with potential neurovascular and bladder injury, more blood loss and it is contraindicated with visceral injury and contaminated field so many surgeons not prefer it [4]. The INFIX is a pelvic subcutaneous cross-over internal fixator technique for fixation of anterior pelvic ring fractures using a Polyaxial pedicle screw anchored to the supra-acetabular region of the ileum in each side and connected by contoured rod which inserted subcutaneously after successful closed reduction. The original description of the INFIX was by Kuttner et al. [3]. The Nickname "INFIX" had been introduced in the English literature in 2012 [5].

\section{Methods}

Our study entails a prospective Cohort Study to evaluate preliminary results of patients with fracture pelvis Tile B \& C from June 2018 to December 2019 treated on orthopedic 
department, Aswan University Hospital and Assiut University Hospital by using INFIX and follow up for one year (mean, 14.5 months). The inclusion criteria were unstable pelvic fracture (type $\mathrm{B} \& \mathrm{C}$ ), recent fracture ( $>10$ days), closed fracture and patient age more than 14 years old. However, the study was excluding obese patients, osteoporotic patients, need for exploration, bladder Injury and unfit for anesthesia.

\section{Material}

sixteen patients were carried out with one year follow up, 15 males (94.75\%) and one female (6.25\%) their age ranges between (17-43 years), Mean (29.54 \pm 13.6$)$. High Energy Trauma was the cause in all cases as expected from the violent nature of pelvic fractures. Motor car accidents was the major cause, it was 9 cases $(56.25 \%), 4$ cases fallen from height (25\%), 2 cases motor bike accident (12.5\%) and one case road traffic accident (6.25\%). According to Tile classification there were 10 case $(62.5 \%)$ pelvic $\mathrm{B}$ fracture and 6 cases $(37.5 \%)$ pelvic $\mathrm{C}$ fracture.

\section{Surgical technique}

If both anterior and posterior fixations are planned, the posterior fixation is typically performed first, then anterior fixation with INFIX. Under general anesthesia, the patient is positioned supine on a flat radiolucent table and the patient's pelvis is widely sterilized and draped. Longitudinal $3 \mathrm{~cm}$ skin incision at the level of anterior inferior iliac spine (AIIS), an obturator outlet view is obtained of one hemi pelvis to mark the incision on the skin, this is achieved by positioning the X-ray tube (source) below and image intensifier (detector) above the table. The detector is tilted $30 \mathrm{o}$ laterally and caudally to obtain a clear "teardrop" appearance of the supra-acetabular bone (Figure 1).

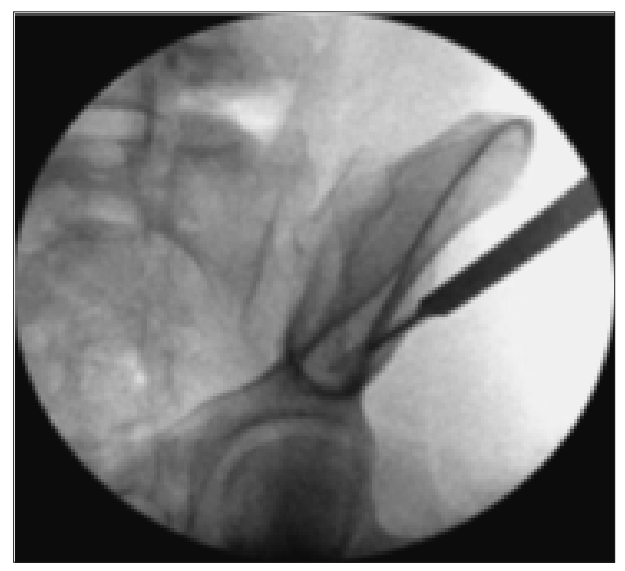

Figure 1: Tear drop, mark skin incision.

Subcutaneous blunt dissection is performed gently to avoid the injury of the lateral femoral cutaneous nerve (LFCN). Then the deep dissection is carried down to the bone in the interval between the sartorius and tensor fasciae lata muscles. The starting point of the track for pedicle screw is created in the anterior inferior iliac spine using the Awl to perforate the near cortex and its position is confirmed radiographically using obturator outlet view (Figure 2).

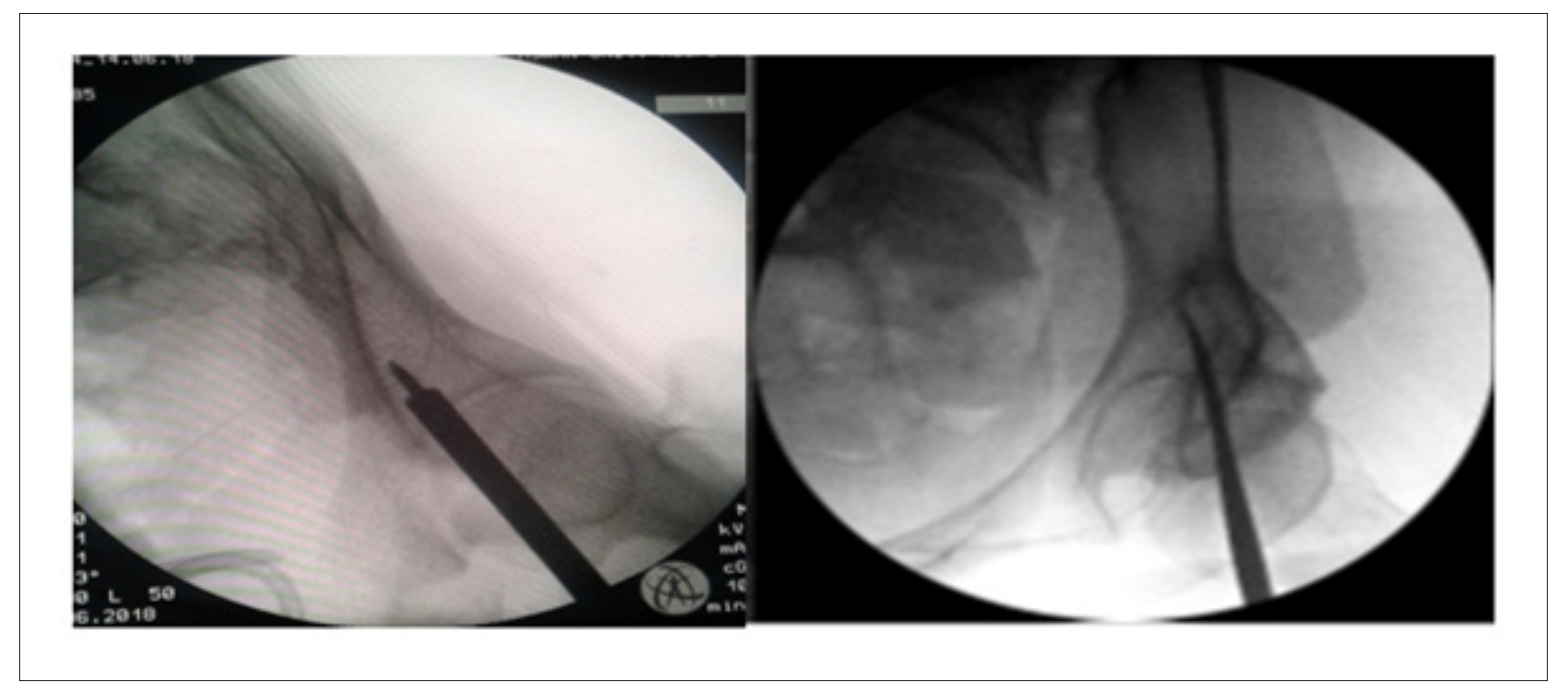

Figure 2: Obturator inlet view, showing awl and pedicle finder insertion.

The awl is then removed and replaced by the pedicle finder which is advanced cranially between the two tables of iliac bone toward the sacroiliac joint and above the level of greater sciatic notch. Two additional fluoroscopic views are obtained. First, an obturator inlet view is obtained by tilting the C-arm detector approximately $30 \mathrm{o}$ laterally and cranially, but fine adjustment should be performed until obtaining a clear view passage of the pedicle finder between well demarcated inner and outer tables of the iliac bone.

The second view, a standard iliac oblique view is obtained by tilting the $\mathrm{C}$-arm detector approximately 300 medially to ensure the starting point has remained at least $2 \mathrm{~cm}$ cranial to the hip to avoid intracapsular placement and that the trajectory of the screw is just cranial to the greater sciatic notch [6]. The pedicle screw is inserted 
in the prepared bony track after removal of the pedicle finder. Screw length is planned to catch enough grip through the strong sciatic buttress region but not reach the sacroiliac joint. In this series, the measurements of the pedicle screws were 6.5 or $7.3 \mathrm{~mm}$ in diameter and $75-100 \mathrm{~mm}$ in length. The screw is inserted until the head is just deep to the level of the fascia lata.
The same procedure is repeated for the contralateral hemi pelvis. Confirmation of the position of pedicular screws: the same three fluoroscopic views (obturator outlet, obturator inlet and iliac) are used to confirm the screw has been advanced along the same prepared track (Figure 3).

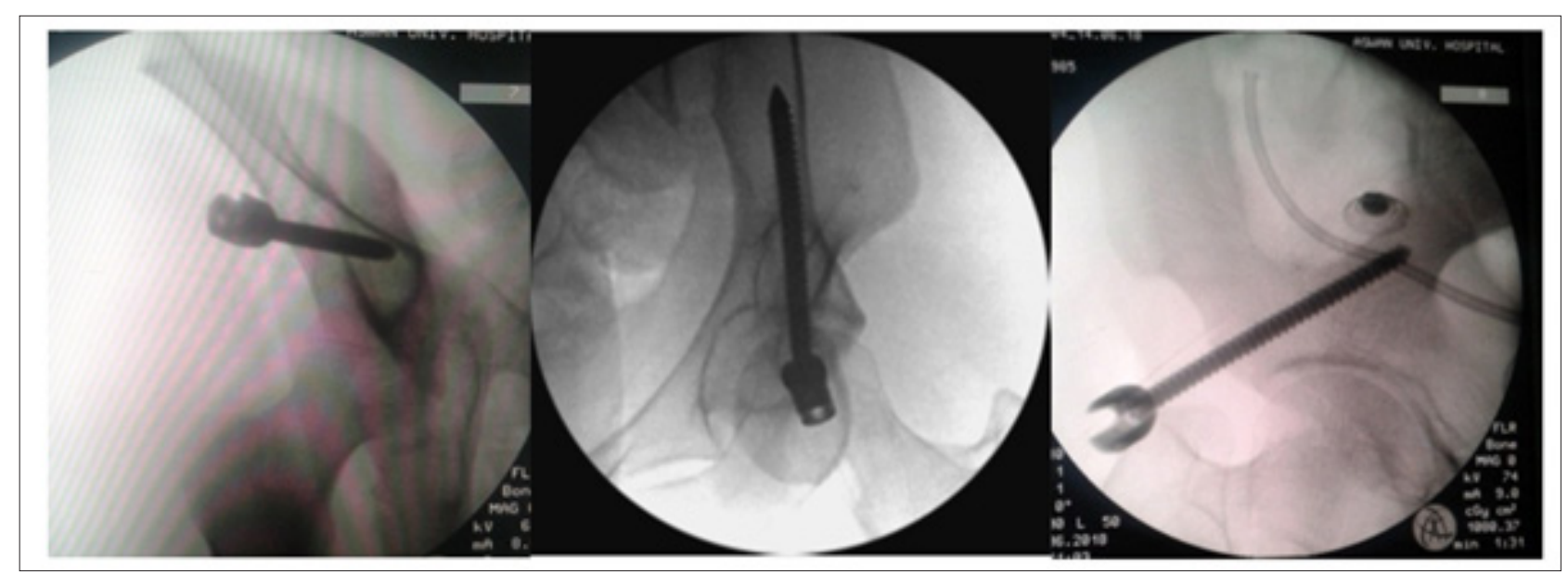

Figure 3: Obturator inlet, obturator outlet, oblique iliac view.

A stainless-steel connecting rod (6mm diameter) is contoured to fit the anatomical contour of inguinal region of the patient without compression the femoral neurovascular bundles or the abdominal viscera. The subcutaneous track for the rode is made by blunt long hemostats that inserted in the subcutaneous tissue at the level of the lower abdominal fold just superficial to the fascia until it reaches the contralateral incision. The bent rod is then inserted in the subcutaneous track and fixed into the screw heads tightly on one side and loose on the other side to permit manipulation during reduction. Multiaxial screw heads with open sections greatly facilitate rod placement. At this point the reduction instruments attached to the screw heads and manipulated according to the requirements of the fracture pattern (compression or distraction device) to obtain fracture reduction. Also, the reduction can be obtained manually through side to side compression of both hemipelvis. With the reduction being held, the assistant tightens the set screws to fix the rod to the screw heads. Then the wounds are closed in layers (Figure 4).

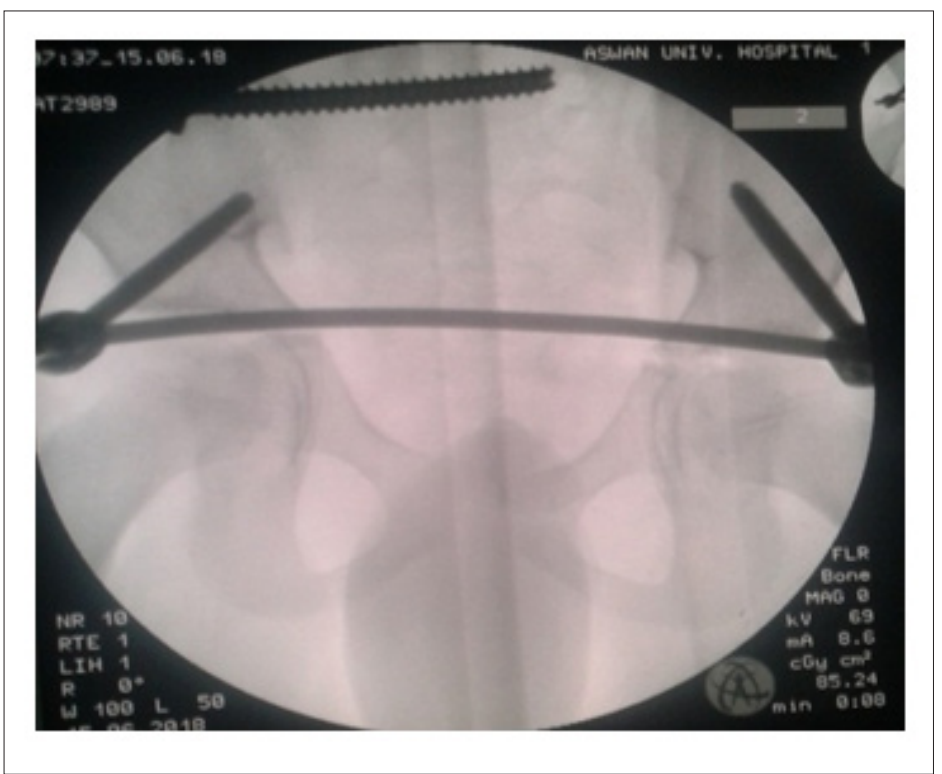

Figure 4: After INFIX application. 


\section{Results}

INFIX application consumes long time at first, this time reduced gradually, so it takes 90 minutes on the first case and 45 minutes on the last case. The mean hospital stays $(7 \pm 4)$ days. Reduction follow up (Matta criteria)[7], 13 cases had excellent reduction postoperative (81.25\%), 2 cases had good reduction $(12.25 \%)$, one case had fair reduction (6.25\%), on follow up after 1.5, 3, 6 and 12 months, the results were the same as early postoperative which indicate stable fixation. MAJEED score [8] of clinical follow up after one year for study group, there were 15 cases of them had excellent (93.75\%) and 1 case had good clinical outcome (6.25\%). Two cases show mild asymptomatic heterotopic ossification (12.5\%), 3 cases had lateral femoral cutaneous nerve irritation postoperative which resolve spontaneously few days postoperative (18.75\%), also one case had DVT after 3 months follow up (6.25\%), no 2ry instability, infection, femoral bundle affection nor technical error.

\section{Discussion}

Many techniques are used for management of unstable fracture pelvis aimed to stabilization of hemodynamic status and fracture reduction. External fixation and plates are the two popular methods used for this issue, each of which has its own complications. External fixation device usually associated with Pin track infection, fixation loosening and difficult nursing [3], on the other hand, Open reduction and internal fixation is associated with potential neurovascular and visceral injury [4]. Recently, the minimally invasive INFIX technique utilized by authors to achieve stable fixation with minimal complication [5]. Our study aimed to evaluate the preliminary result of INFIX technique for treatment of unstable fracture pelvis.

In our study, within the postoperative first day, all patients were satisfied as they were pain free, starting rehabilitation without weight bearing, changing their bed positions even in prone decubitus without limitations. Patients started protected weight bearing after 6 weeks and full weight bearing after 12 weeks. Although, some patients could feel the implants, it did not interfere with their ability to ambulate, this is the major advantages of INFIX which reported on other studies [5].

Clinically and radiologically, fracture union rate was $100 \%$ (mean union time was 10 weeks) without any union complication although some cases of delayed union reported on other literatures [4]. The authors used polyaxial screws vary from $7-10 \mathrm{~mm}$ in diameter [3,5,9]. We used two screws diameter; 6.5 and $7.3 \mathrm{~mm}$ which achieved an excellent stability. The largest screw diameter may contribute to LFCN neuropraxia because Scheyerer reported no LFCN palsies using a smaller screw [10]. In our study, unilateral lateral femoral cutaneous nerve (LFCN) irritation was reported only in three cases $(18.75 \%)$ which improved spontaneously few days later. Rahul et al, reported irritation of the lateral femoral cutaneous nerve in 27 of the 91 patients (30\%) [11]. The mean operation time for INFIX application almost equal to the time reported in other literatures which was 51 min (range 44-65) [3].

As regard to infection, loss of reduction, heterotopic ossification, DVT, LFCN and femoral nerve palsy, our final results were better than other studies [11] (Table 1).

Table 1: Our results compared to other studies.

\begin{tabular}{|c|c|c|}
\hline & Our Study & Other Studies [8] \\
\hline Infection & $0 \%$ & $3.92 \%$ \\
\hline Loss of reduction & $0 \%$ & $7 \%$ \\
\hline Heterotopic ossification & $12.5 \%$ & $35 \%$ \\
\hline DVT & $6.25 \%$ & $0 \%$ \\
\hline LFCN & $18.75 \%$ & $30 \%$ \\
\hline femoral nerve palsy & $0 \%$ & $9 \%$ \\
\hline
\end{tabular}

Table 2: Our results compared to external fixator.

\begin{tabular}{|c|c|c|}
\hline & EX.FIX & INFIX \\
\hline Infection & $35 \%$ & $0 \%$ \\
\hline Loss of reduction & $12 \%$ & $0 \%$ \\
\hline revision & $17 \%$ & $0 \%$ \\
\hline Nursing & Difficult & easy \\
\hline
\end{tabular}

Table 3: Our results compared to plates and screws.

\begin{tabular}{|c|c|c|}
\hline & Plate & INFIX \\
\hline Dissection & Marked & limited \\
\hline Blood loss & More $(600 \mathrm{ml})$ & Less $(300 \mathrm{ml})$ \\
\hline Time of Op. & Longer & Shorter \\
\hline Complications rate & More $(28.6 \%)$ & Less $(14.3 \%)$ \\
\hline
\end{tabular}


In our study INFIX Achieve better results than the studies $[12,13]$ using the external fixator (Table 2) and better than the studies of internal fixation using plates and screws [14] for management of unstable fracture pelvis (Table 3). Larger sample studies with longer follow up are needed.

\section{Conclusion}

INFIX achieved better results than other fixation methods used for management of unstable fracture pelvis as regard to fracture reduction, stability and bone healing with less operative time, small incision and less complication rate.

\section{References}

1. Gansslen A, Pohlemann T, Paul C, Lobenhoffer P, Tscherne H (1996) Epidemiology of pelvic ring injuries. Injury 27 Suppl 1: S-A13-20.

2. Tile M, Helfet DL, Kellam JF, Vrahas M (2015) Fractures of the pelvis and acetabulum: Principles and methods of management. ( $4^{\text {th }}$ edn), New York, USA.

3. Wayne H, Andrew B, James W, Edward B, Rodney J, et al. (2016) A prospective case series for a minimally invasive internal fixation device for anterior pelvic ring fractures. Journal of Orthopaedic Surgery and Research 11(1): 135 .

4. Peter AC, Evgeny AD, Jeffrey AG (2015) Minimally invasive fixation for anterior pelvic ring disruptions. Injury 46 Suppl 3: S27-S34.

5. Vaidya R, Colen R, Vigdorchik J, Tonnos F, Sethi A (2012) Treatment of unstable pelvic ring injuries with an internal anterior fixator and posterior fixation: initial clinical series. J Orthop Trauma 26(1): 1-8.

6. Gardner MJ, Mehta S, Mirza A, Ricci WM (2010) Anterior pelvic reduction and fixation using a subcutaneous internal fixator. J Orthop Trauma 26(5): 314-321.

7. Matta JM, Tornetta $P$ (1996) Internal fixation of unstable pelvic ring injuries. Clin Orthop Relat Res 329: 129-140.

8. Majeed SA (1990) External fixation of the injured pelvis, the functional outcome. J Bone Joint Surg Br 72(4): 612-614.

9. Owen MT, Tinkler B, Stewart R (2013) Failure and salvage of INFIX instrumentation for pelvic ring disruption in a morbidly obese patient. J Orthop Trauma 27(10): e243-246.

10. Scheyerer MJ, Zimmermann SM, Osterhoff G, Tiziani S, Simmen HP, et al. (2014) Anterior subcutaneous internal fixation for treatment of unstable pelvic fractures. BMC Res Notes 7: 133.

11. Rahul V, Erik NK, Patrick FB, Derek GD, Ren JC, et al. (2012) Complications of anterior subcutaneous internal fixation for unstable pelvis fractures. Clin Orthop Relat Res 470(8): 2124-2131.

12. Scaglione M, Parchi P, Digrandi G, Latessa M, Guido G (2010) External fixation in pelvic fractures. Musculoskelet Surg 94(2): 63-70.

13. Arazi M, Kutlu A, Mutlu M, Yel M, Kapiciglu MI (2000) The pelvic external fixation: the mid-term results of 41 patients treated with a newly designed fixator. Arch Orthop Trauma Surg 120(10): 584-586.

14. Yingchao Y, Junhao L, Ruipeng Z, Shilun L, Zhenqing J, et al. (2019) Anterior subcutaneous internal fixator (INFIX) versus plate fixation for pelvic anterior ring fracture. Scientific Reports 9: 2578. 